\title{
Processus hydrogéochimiques et origine des sources naturelles dans le degré carré de Daloa (Centre ouest de la Côte d'Ivoire)
}

\author{
Raymond LIGBAN ${ }^{1}$, Lanciné Droh GONE ${ }^{1 *}$, Bamory KAMAGATE ${ }^{1}$, \\ Mahaman Bachir SALEY ${ }^{2}$ et Jean BIEMI ${ }^{2}$ \\ ${ }^{1}$ Laboratoire Géosciences et Environnement, Université d'Abobo-Adjamé, 02 BP 801 Abidjan 02, \\ Côte d'Ivoire. \\ ${ }^{2}$ Laboratoire des Sciences et Techniques de l'Eau et de l'Environnement, Université de Cocody, 22 BP 582 \\ Abidjan 22, Côte d'Ivoire. \\ *Auteur correspondant, E-mail: dialou25_9@yahoo.fr
}

\section{RESUME}

L'étude des processus hydrogéochimiques des aquifères du degré carré de Daloa à travers la caractérisation des paramètres physico-chimiques et l'analyse des équilibres eau-minéraux a permis de déterminer l'origine des sources naturelles de cette région. Les eaux sont dans l'ensemble acides et faiblement minéralisées. La signature chimique des eaux de sources marquée par de faibles conductivités se rapproche nettement de celle des eaux de puits qui captent les altérites, et s'éloigne de celle des eaux de forages qui interceptent le socle fissuré. Le calcul des indices de saturation par rapport à la dolomie et la calcite, et l'utilisation du diagramme de korjinski montre que les eaux de source et de puits se caractérisent par une faible minéralisation traduisant un bref temps de séjour, contrairement aux eaux de forages plus minéralisées et donc plus anciennes. Ces caractéristiques hydrogéochimiques suggèrent que les sources naturelles du degré carré de Daloa sont des exutoires de nappes d'altérites.

(C) 2009 International Formulae Group. All rights reserved.

Mots clés : Nappes d'altérites et de fissures, émergence, minéralisation, altération, lessivage.

\section{INTRODUCTION}

Les sources sont des émergences naturelles de nappes qui apparaissent d'une manière localisée ou diffuse à la surface du sol. Elles constituent des zones de vidange naturelle ou des exutoires ponctuels d'eaux souterraines. Celles-ci sont dans de nombreuses régions, captées à l'émergence pour l'alimentation en eau des populations (Bourlier et al., 2005; Karakaya, 2006; Omar, 2008). La part des sources dans la consommation mondiale des ressources en eau est estimée à $6,3 \%$ (Oi-Wah et Shin-Fai, 2002).

Sur la base de l'origine, des conditions d'émergence et des caractéristiques physico- chimiques, on peut distinguer les sources thermales, les sources minérales (Canellas, 1995 ) et les sources thermominérales généralement rencontrées en régions volcaniques (Virgilio et Zilda, 2006). L'origine de ces émergences est souvent liée à des remontées de nappes profondes qui apparaissent par action de l'érosion entrainant l'affleurement des fractures, des flexures ou des failles à la surface du sol (Pouchan, 1995 ; Favara et al., 1998).

Outre ces émergences de nappes profondes, il est fait état dans la littérature (Faillat et Grillot, 1993; Bourlier et al., 2005) d'émergences correspondant aux exutoires de nappes superficielles, soit $\mathrm{du}$ fait de 
formations imperméables sous-jacentes, soit $\mathrm{du}$ fait de la topographie. Ces eaux sont caractérisées par une faible minéralisation (Faillat et Grillot, 1993) et se rencontrent généralement dans les zones de socle cristallin et cristallophyllien à forte pente. On les observe en particulier dans les zones de socle granitique où les sources constituent pour la plupart, des exutoires de nappes qui suivent généralement la topographie.

Dans le degré carré de Daloa situé au Centre-Ouest de la Côte d'Ivoire, les sources naturelles constituent l'un des principaux points d'accès à la ressource en eau pour les populations majoritairement rurales. Leur abondance dans cette région fait la particularité du Centre-Ouest de la Côte d'Ivoire. Les travaux de Maillary (1964) dans le cadre de l'élaboration de la carte hydrogéologique de la Côte d'Ivoire ont permis de dénombrer 98 sources dont $86,73 \%$ sont pérennes. Hormis ce travail d'inventaire, l'origine et les caractéristiques physicochimiques de ces sources n'ont pas encore été abordées dans la littérature. Elles restent de ce point de vue peu connues au plan hydrogéologique alors qu'elles constituent une source potentielle d'alimentation en eau des populations.

L'objectif visé dans cette étude est de mieux connaître l'origine des sources naturelles du degré carré de Daloa à partir de l'étude hydrogéochimique des réservoirs souterrains et des sources à l'émergence. Il s'agit d'une part de rechercher les caractéristiques physico-chimiques majeures des eaux de sources et d'autre part de connaître leur origine sur la base de la différenciation hydrochimique des différents aquifères. Pour atteindre cet objectif, des analyses géochimiques des roches et des analyses physico-chimiques d'eau de sources, d'eau d'altérites (puits) et d'eau de socle (forages) ont été effectuées.

\section{MATERIEL ET METHODES \\ Site d'étude}

Le degré carré de Daloa, avec une superficie de $10000 \mathrm{~km}^{2}$, se situe au Centre Ouest de la Côte d'Ivoire entre les latitudes $6^{\circ}$ et $7^{\circ}$ Nord et les longitudes $6^{\circ}$ et $7^{\circ}$ Ouest (Figure 1). Le relief de la région est caractérisé par un abaissement de direction NE-SO. Les altitudes évoluent entre $149 \mathrm{~m}$ et $382 \mathrm{~m}$.

Le climat de la région de Daloa est de type tropical humide de transition. Il est caractérisé par une saison sèche allant d'octobre à mars et une saison des pluies ayant deux maxima, l'un en juin et l'autre en septembre. Les saisons sèches et humides alternent avec des températures variant de $24,65^{\circ} \mathrm{C}$ à $27,75^{\circ} \mathrm{C}$ en moyenne (Figure 2). Avec des pluies annuelles qui sont passées de $1868,5 \mathrm{~mm}$ en 1968 à $1120,4 \mathrm{~mm}$ en 2005 , la région connaît une baisse pluviométrique de l'ordre de $40 \%$.

Le substratum géologique du degré carré de Daloa est caractérisé par les formations du Précambrien moyen. Ces formations sont dominées par les granites, les migmatites et les schistes. Les principaux minéraux rencontrés dans ces roches sont le quartz, les micas, la muscovite, la biotite, les amphiboles, l'albite et la magnétite. De façon accessoire, on observe l'apatite, le microcline, le zircon et l'orthose (Papon, 1973).

L'altération de ces formations conduit à la mise en place d'un recouvrement altéritique d'épaisseur variable capté par les puits villageois. Plus en profondeur, se trouve le socle cristallin fissuré capté généralement par les forages d'hydraulique villageoise (Lasm et al., 2004).

\section{Méthodologie}

Une campagne d'échantillonnage des eaux s'est effectuée en mars 2008; elle a porté sur 42 sources qui s'observent pour la plupart au bas des versants, 10 puits à grand diamètre de profondeur moyenne $8 \mathrm{~m}$ et 14 forages (de profondeur moyenne $70 \mathrm{~m}$ ) équipés par des pompes à motricité humaine.

La température, le $\mathrm{pH}$, la conductivité électrique ont été mesurés sur le terrain à l'aide d'un multimètre de marque WWT82362. L'oxygène dissous et la turbidité ont également été mesurés sur le terrain respectivement avec un oxymètre Crison Oxi 330 et un turbidimètre de marque Hach 2100 P. Les ions hydrogénocarbonates ont été déterminés par titration avec l'acide chlorhydrique (ISO 9963-2/1994 (F)). 


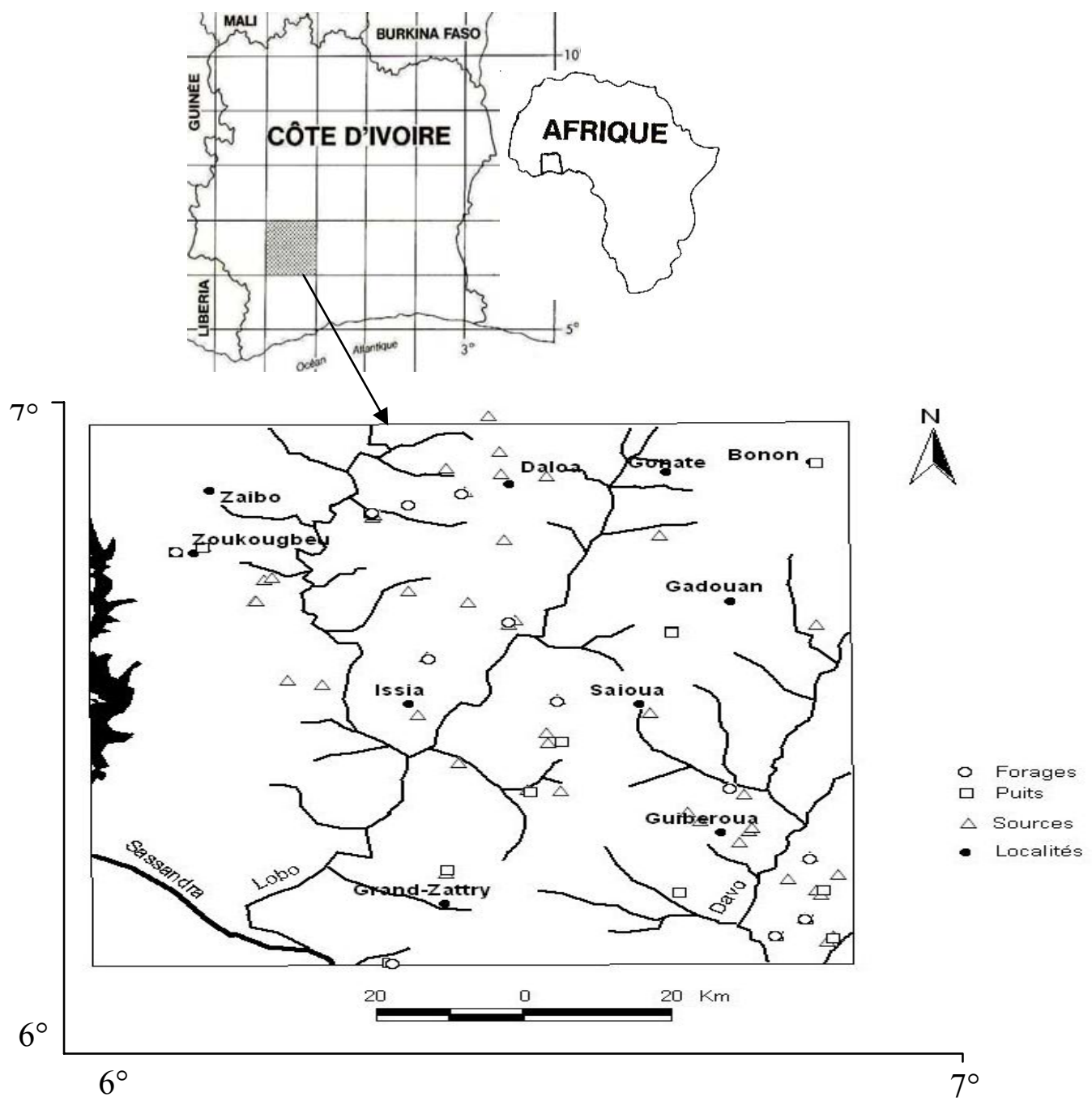

Figure 1: Situation géographique du degré carré de Daloa et localisation des points d'échantillonnage.

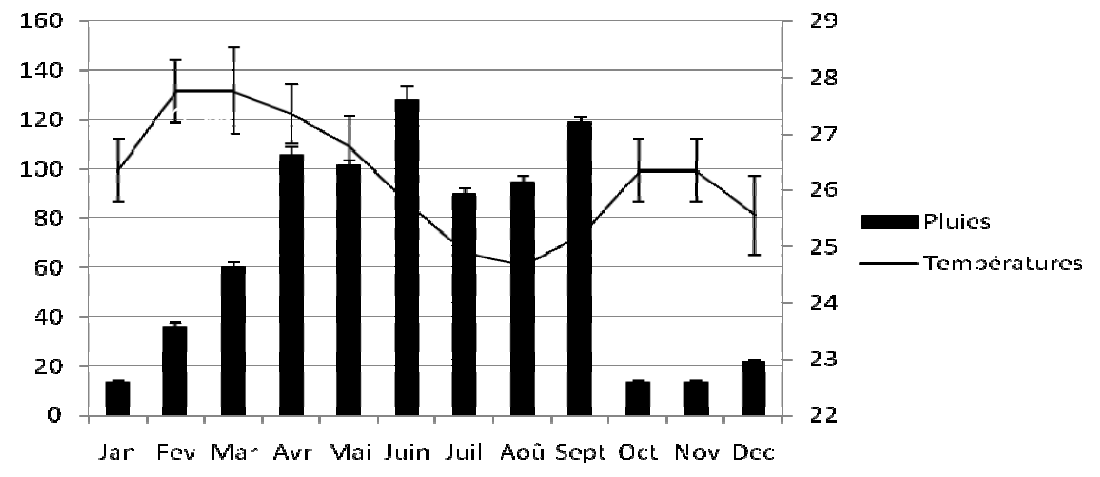

Figure 2 : Pluies moyennes et températures moyennes mensuelles (1979-2006). 
Les cations $\left(\mathrm{Ca}^{2+}, \mathrm{Mg}^{2+}, \mathrm{Na}^{+}, \mathrm{K}^{+}, \mathrm{Fe}^{2+}\right)$ et anions $\left(\mathrm{Cl}^{-}, \mathrm{NO}_{3}{ }^{-}, \mathrm{PO}_{4}{ }^{3-}, \mathrm{SO}_{4}{ }^{2-}\right)$ ont été analysés par chromatographie ionique après filtration des échantillons sur membrane en cellulose de porosité $0,45 \mu \mathrm{m}$. Les colonnes CS-12 et AS4A-SC ont été utilisées respectivement pour l'analyse des cations et des anions. Outre les analyses d'eau, des données géochimiques de roches ont été collectées à partir des bulletins d'analyses réalisées dans le cadre des missions de levés géologiques.

Les conductivités électriques et les hydrofaciès ont été utilisés pour mettre en évidence la différenciation chimique entre les eaux des différents aquifères. Ces paramètres ont déjà été utilisés comme marqueur dans des études similaires (Grillot et al., 1990; Kamagaté, 2006). Le calcul des indices de saturation par rapport à la calcite et la dolomie et l'analyse des diagrammes de stabilité eauminéraux ont permis d'aborder le fonctionnement hydrogéochimique des aquifères.

\section{RESULTATS}

\section{Caractéristiques géochimiques des roches}

Le tableau 1 montre les teneurs en éléments chimiques des différentes roches. D'une manière générale les teneurs en $\mathrm{SiO}_{2}$ sont les plus importantes. Elles sont dans l'ensemble supérieures à $60,00 \%$. Outre $\mathrm{SiO}_{2}$, la proportion en $\mathrm{Al}_{2} \mathrm{O}_{3}$ est relativement élevée dans toutes les roches avec une contribution maximale de $18,75 \%$. Les alcalins sont dominés par $\mathrm{NaO}$ avec des teneurs qui varient de $0,32 \%$ à $7,85 \%$. En revanche, les alcalinoterreux dont $\mathrm{CaO}$ est l'élément dominant ont des valeurs qui restent inférieures à 5,00\%. Les fortes proportions en silice et alcalins contrairement aux alcalino-terreux dont les contributions restent faibles montrent que ces roches sont acides.

\section{Caractéristiques physico-chimiques des eaux}

Les concentrations moyennes des paramètres physico-chimiques analysés sont reportées dans le tableau 2. Les températures enregistrées, avec des moyennes de $25,04{ }^{\circ} \mathrm{C}$, $24,87{ }^{\circ} \mathrm{C}, 25,16{ }^{\circ} \mathrm{C}$ respectivement dans les sources, puits et forages, sont sensiblement similaires. Les turbidités moyennes des eaux de sources $(4,85 \mathrm{NTU})$ et de puits $(4,68 \mathrm{NTU})$ sont similaires et nettement plus élevées que celles des eaux de forages (0,68 NTU).

Les eaux sont dans l'ensemble acides, avec une acidité plus marquée pour les sources et les puits.

L'ensemble des eaux échantillonnées apparaît peu minéralisé avec des conductivités généralement inférieures à $165 \mu \mathrm{S} / \mathrm{cm}$. Cette faible minéralisation est plus marquée pour les eaux de sources et de puits qui présentent des conductivités moyennes respectives de 51,18 $\pm 20,20 \mu \mathrm{S} / \mathrm{cm}$ et $77,91 \pm 45,82 \mu \mathrm{S} / \mathrm{cm}$ contre $110,11 \pm 35,82 \mu \mathrm{S} / \mathrm{cm}$ dans les forages.

Tableau 1 : Teneurs (\%) des éléments chimiques dans les roches.

\begin{tabular}{lcccccccc}
\hline Roches & $\mathrm{SiO}_{2}$ & $\mathrm{Al}_{2} \mathrm{O}_{3}$ & $\mathrm{Fe}_{2} \mathrm{O}_{3}$ & $\mathrm{MnO}$ & $\mathrm{MgO}$ & $\mathrm{CaO}$ & $\mathrm{Na}_{2} \mathrm{O}$ & $\mathrm{K}_{2} \mathrm{O}$ \\
\hline Granophyte & 61,40 & 15,60 & 3,20 & 0,16 & 4,75 & 2,20 & 7,85 & 0,35 \\
Schiste greseux & 66,99 & 15,64 & 3,43 & 0,04 & 2,07 & 3,24 & 3,65 & 1,35 \\
Schistes sericiteux & 64,20 & 18,75 & 6,55 & 0,05 & 0,50 & 1,65 & 3,15 & 0,85 \\
Micaschiste & 71,30 & 13,50 & 3,90 & 0,05 & 1,55 & 1,05 & 2,45 & 2,20 \\
Métarhyolite & 76,90 & 11,58 & 1,96 & 0,18 & 0,12 & 0,67 & 5,70 & 1,80 \\
Granodiorite & 64,96 & 15,14 & 0,83 & 0,05 & 2,18 & 2,69 & 4,90 & 4,20 \\
Granite à biotite & 69,76 & 14,13 & 0,53 & 0,03 & 0,68 & 3,86 & 4,70 & 3,50 \\
Granite à 2 micas & 72,05 & 14,89 & 0,71 & 0,02 & 0,40 & 1,28 & 3,70 & 5,15 \\
\hline
\end{tabular}


Tableau 2 : Caractéristiques physico-chimiques moyennes des eaux échantillonnées.

\begin{tabular}{|c|c|c|c|c|c|c|c|c|c|c|}
\hline & & & ource (n & $=42)$ & & Pui & $\bar{s}(n=10)$ & & orages (n & $=14)$ \\
\hline & & Min & Max & Moy $\pm \sigma$ & Min & Max & Мoy $\pm \sigma$ & Min & Max & $\operatorname{Moy} \pm \sigma$ \\
\hline $\mathrm{T}$ & $\left({ }^{\circ} \mathrm{C}\right)$ & 23,80 & 26,50 & $25,04 \pm 0,67$ & 24,10 & 26,10 & $24,87 \pm 0,56$ & 24,10 & 26,00 & $25,16 \pm 0,62$ \\
\hline Turb & (NTU) & 0,17 & 22,71 & $4,85 \pm 5,57$ & 0,26 & 22,35 & $4,68 \pm 6,90$ & 0,13 & 2,68 & $0,68 \pm 0,81$ \\
\hline $\mathrm{pH}$ & & 4,14 & 6,06 & $4,69 \pm 0,37$ & 4,60 & 5,80 & $4,75 \pm 0,57$ & 4,55 & 6,06 & $5,51 \pm 0,38$ \\
\hline Eh & $(\mathrm{mV})$ & 37,00 & 155,00 & $121,21 \pm 22,46$ & 55,00 & 185,00 & $117,60 \pm 33,73$ & 37,00 & 129,00 & $71,43 \pm 22,97$ \\
\hline Cond & $(\mu \mathrm{S} / \mathrm{cm})$ & 21,40 & 103,30 & $51,18 \pm 20,2$ & 33,30 & 159,30 & $77,91 \pm 45,82$ & 32,00 & 164,90 & $110,11 \pm 35,82$ \\
\hline $\mathrm{O}_{2}$ & $(\mathrm{mg} / \mathrm{L})$ & 0,98 & 7,94 & $2,70 \pm 1,31$ & 1,72 & 4,39 & $3,07 \pm 0,86$ & 1,88 & 4,89 & $2,94 \pm 0,91$ \\
\hline $\mathrm{Na}^{+}$ & $(\mathrm{mg} / \mathrm{L})$ & 1,52 & 14,90 & $6,25 \pm 2,78$ & 1,81 & 90,46 & $22,17 \pm 33,18$ & 0,41 & 17,48 & $7,54 \pm 4,67$ \\
\hline $\mathrm{K}^{+}$ & $(\mathrm{mg} / \mathrm{L})$ & 0,29 & 5,95 & $2,08 \pm 1,20$ & 0,90 & 7,55 & $2,21 \pm 2,03$ & 0,77 & 4,98 & $2,69 \pm 1,40$ \\
\hline $\mathrm{Ca}^{2+}$ & $(\mathrm{mg} / \mathrm{L})$ & 0,00 & 3,83 & $1,18 \pm 0,89$ & 0,00 & 3,10 & $1,36 \pm 1,11$ & 0,35 & 6,41 & $2,93 \pm 1,77$ \\
\hline $\mathrm{Mg}^{2+}$ & $(\mathrm{mg} / \mathrm{L})$ & 0,04 & 4,65 & $0,61 \pm 0,74$ & 0,05 & 2,76 & $0,61 \pm 0,82$ & 0,24 & 5,34 & $1,92 \pm 1,67$ \\
\hline $\mathrm{Cl}^{-}$ & $(\mathrm{mg} / \mathrm{L})$ & 2,75 & 17,63 & $6,85 \pm 3,58$ & 3,02 & 24,65 & $11,43 \pm 6,05$ & 4,13 & 13,31 & $7,43 \pm 2,46$ \\
\hline $\mathrm{SO}_{4}{ }^{2-}$ & $(\mathrm{mg} / \mathrm{L})$ & 0,09 & 6,00 & $0,87 \pm 1,08$ & 0,06 & 1,36 & $0,98 \pm 0,44$ & 0,19 & 5,00 & $1,63 \pm 1,87$ \\
\hline $\mathrm{Fe}^{2+}$ & $(\mathrm{mg} / \mathrm{L})$ & 0,00 & 6,10 & $0,34 \pm 0,92$ & 0,02 & 6,10 & $0,73 \pm 1,89$ & 0,01 & 1,53 & $0,28 \pm 0,43$ \\
\hline $\mathrm{HCO}_{3}$ & $(\mathrm{mg} / \mathrm{L})$ & 6,10 & 21,35 & $8,84 \pm 3,42$ & 6,10 & 7,93 & $7,02 \pm 0,72$ & 34,77 & 67,10 & $46,01 \pm 9,56$ \\
\hline $\mathrm{NO}_{3}^{-}$ & $(\mathrm{mg} / \mathrm{L})$ & 0,65 & 18,60 & $5,99 \pm 4,84$ & 0,01 & 42,01 & $10,35 \pm 11,70$ & 4,83 & 25,25 & $10,38 \pm 5,00$ \\
\hline $\mathrm{NH}_{4}^{+}$ & $(\mathrm{mg} / \mathrm{L})$ & 0,00 & 0,72 & $0,09 \pm 0,14$ & 0,00 & 0,84 & $0,14 \pm 0,26$ & 0,00 & 0,12 & $0,02 \pm 0,03$ \\
\hline $\mathrm{PO}_{4}{ }^{3-}$ & $(\mathrm{mg} / \mathrm{L})$ & 0,00 & 2,18 & $0,96 \pm 0,64$ & 0,30 & 13,40 & $2,51 \pm 3,88$ & 0,00 & 3,15 & $1,74 \pm 1,11$ \\
\hline
\end{tabular}

Min $=$ minimum; Max $=$ maximum; Moy $=$ moyenne $\sigma=$ écart-type

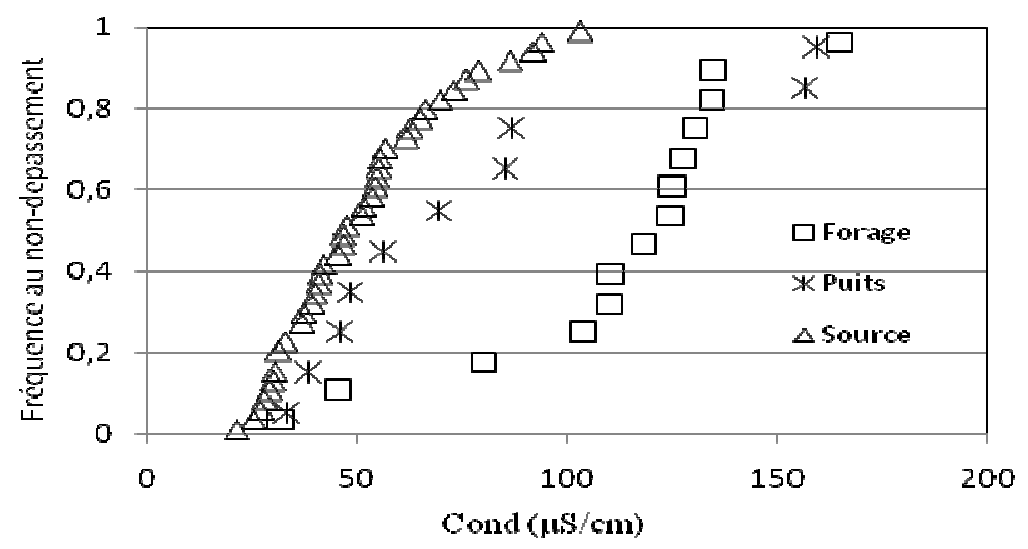

Figure 3: Fréquence au non-dépassement des conductivités des différents échantillons prélevés.

Les fréquences au non-dépassement des conductivités (Figure 3) montrent dans l'ensemble une nette distinction entre les différents types d'eaux. Elles indiquent que $50 \%$ des échantillons de sources, puits et forages ont des conductivités respectivement inférieures à $50 \mu \mathrm{S} / \mathrm{cm}, 100 \mu \mathrm{S} / \mathrm{cm}$ et 150 $\mu \mathrm{S} / \mathrm{cm}$.

Le faciès chloruré nitraté sodipotassique domine la majorité des eaux de sources et puits (Figure 4). En revanche dans les forages, le faciès bicarbonaté est dominant. 


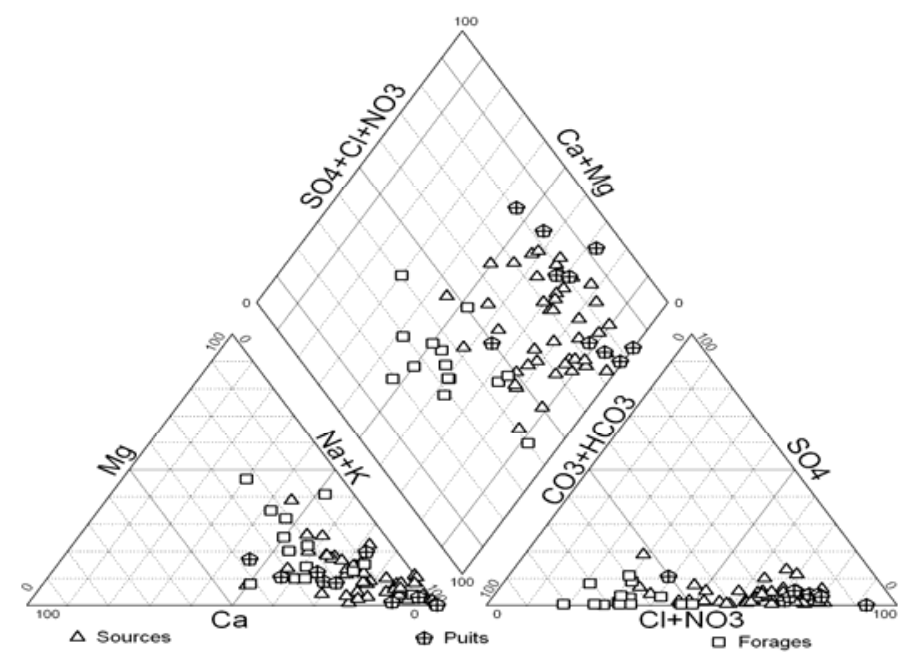

Figure 4 : Représentation des différents échantillons dans le diagramme de Piper.

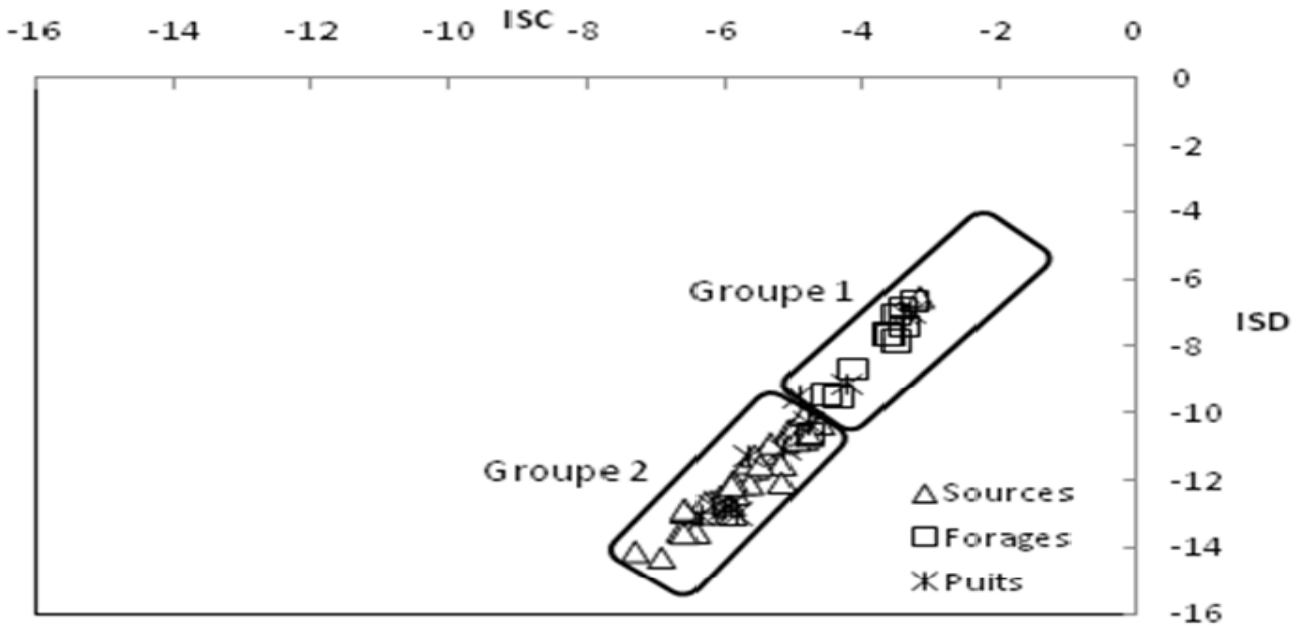

Figure 5: Evolution des échantillons dans le diagramme ISD $=\mathrm{f}(\mathrm{ISC})$.

\section{Equilibre eau-minéraux dans les aquifères}

L'évolution des indices de saturation par rapport à la dolomie (ISD) en fonction des indices de saturation par rapport à la calcite (ISC) (Figure 5) montre que les eaux sont sous-saturées vis-à-vis de ces deux minéraux. Toutefois, deux groupements d'eau se dégagent et permettent une distinction entre les différents réservoirs échantillonnés.

Le groupe caractérisé par des indices de saturation relativement plus élevés est essentiellement dominé par les eaux de forage.
En revanche, le second groupe caractérisé par des indices de saturation plus faibles est composé d'eaux de puits et de sources. Cette disposition des échantillons d'eau dans le diagramme ISC - ISD indique que les eaux de sources et de puits ont un bref temps de séjour dans leur aquifère par rapport aux eaux de forage. Aussi, la position rapprochée des échantillons de sources et de puits permet-elle de supposer l'existence probable d'un échange entre ces deux systèmes. 
Dans le diagramme de Korjinski, les eaux appartiennent aux domaines de stabilité de la kaolinite, de la gibbsite, et de la $\mathrm{Ca}$ montmorillonite (Figure 6).

Les échantillons provenant des sources et puits se situent majoritairement dans le domaine de la kaolinite (Figure 6a). Leur état d'équilibre avec la kaolinite et la gibbsite est caractéristique de leurs faibles teneurs en silice. Ces teneurs montrent que le phénomène d'hydrolyse à l'origine de la minéralisation des eaux souterraines se réalise partiellement dans ces aquifères, ce qui justifie leur faible minéralisation (faibles conductivités) résultant de leur bref temps de contact avec l'encaissant plus lessivé et/ou drainé. En revanche, la majorité des échantillons de forages se situent dans le domaine de stabilité de la Camontmorillonite ou proche de celui du Namontmorillonite qui sont des minéraux plus solubles que la kaolinite. Dans ce cas, le phénomène d'hydrolyse des minéraux primaires enrichi les eaux en silice dissoute et en cations rapprochant la solution de l'équilibre avec ces minéraux de néoformation (montmorillonite). Les eaux de forage sont donc caractérisées par un temps de contact plus long avec l'encaissant en accord avec les résultats précédents.
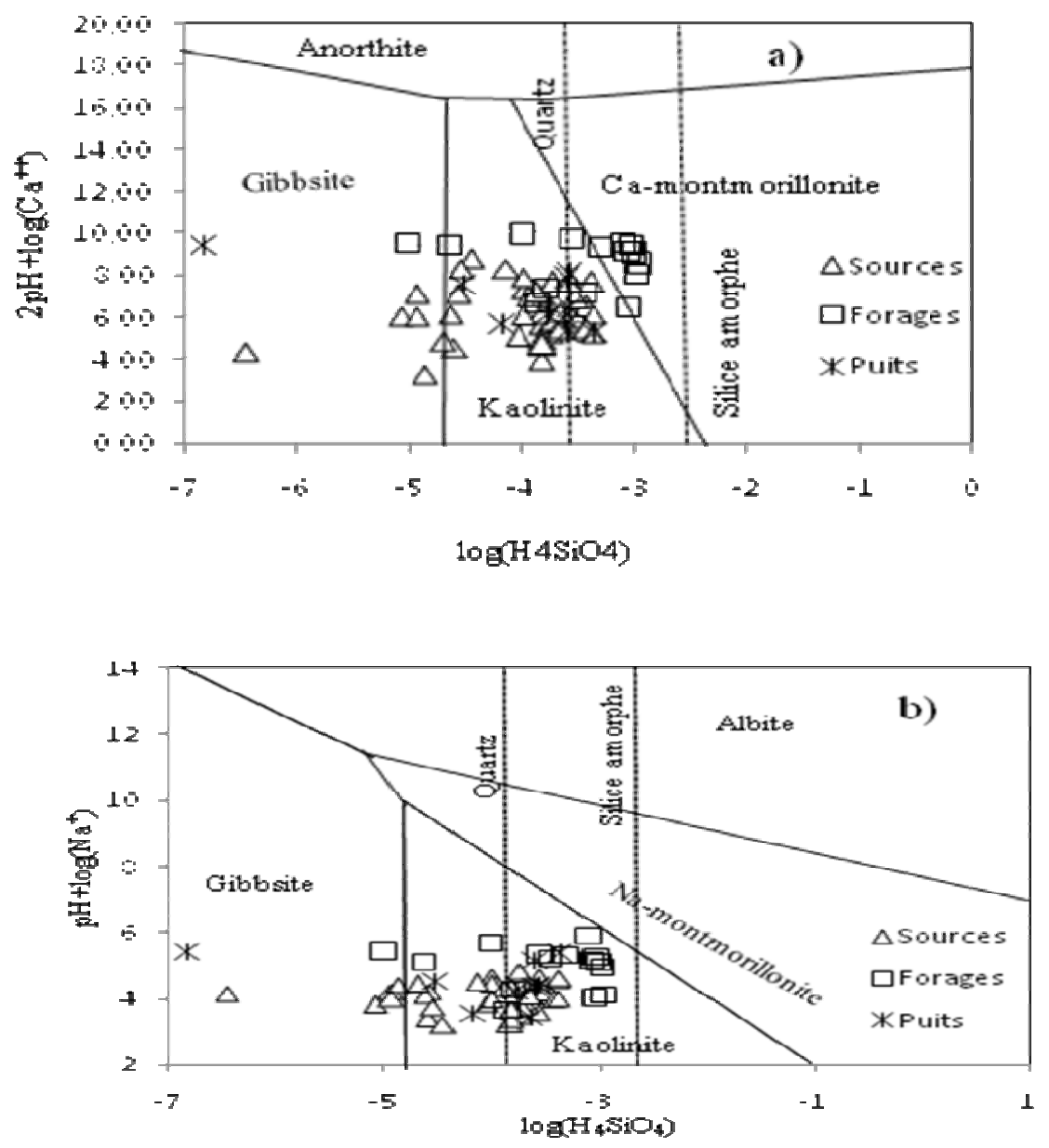

Figure 6 : Equilibre entre phase solide et phase aqueuse: domaine de stabilité du système $\mathrm{CaO}-\mathrm{Al}_{2} \mathrm{O}_{3}-\mathrm{SiO}_{2}-\mathrm{H}_{2} \mathrm{O}$ et $\mathrm{Na}_{2} \mathrm{O}-\mathrm{Al}_{2} \mathrm{O}_{3}-\mathrm{SiO}_{2}-\mathrm{H}_{2} \mathrm{O}$. 


\section{DISCUSSION}

Les eaux souterraines du degré carré de Daloa sont acides et faiblement minéralisées en accord avec le caractère acide des roches encaissantes $\left(\mathrm{SiO}_{2}>60 \%\right)$. Cette acidité, du fait de l'abondance du quartz, confère aux roches une résistance aux phénomènes d'hydrolyse responsable de la minéralisation des eaux souterraines (Boukari, 1982). Dans ces conditions, l'acide carbonique issue de l'hydratation du $\mathrm{CO}_{2}$ persiste et confère à l'eau son acidité (Schoeller, 1980). L'acidité et la faible minéralisation des eaux souterraines de socle en Côte d'Ivoire ont déjà été signalées par Goné et al. (2004) travaillant sur les caractéristiques physico-chimiques majeures des eaux des aquifères fissurés de la région d'Agboville (Sud-Est de la Côte d'Ivoire).

Par ailleurs, la minéralisation des eaux est plus prononcée dans les forages que dans les puits et sources. Cette différenciation traduit un temps de résidence plus prolongé pour les eaux de forage, plus profondes (Faillat et Grillot, 1993 ; Poulichet et al, 2002) par rapport aux eaux de puits et sources.

Le faciès bicarbonaté des eaux de forages à la différence des eaux de sources et puits qui sont chlorurées nitratées montre également l'existence d'une différenciation chimique entre les nappes captées. Le faciès bicarbonaté est une caractéristique majeure des eaux de socle en milieu cristallin et cristallophyllien. Des travaux effectués dans d'autres régions de la Côte d'Ivoire en milieu de socle notamment dans la région de Man (Goné et al., 2005) et dans la zone de Soubré (Soro, 2002) mettent également en évidence l'importance du faciès bicarbonaté. Les ions bicarbonates proviennent principalement de l'hydrolyse acide des roches. Par exemple l'anorthite (plagioclase du pôle calcique) produit de la kaolinite et libère du calcium et des ions bicarbonates selon la réaction:

$$
\begin{array}{ll}
\mathrm{CaAl}_{2} \mathrm{Si}_{2} \mathrm{O}_{8}+3 \mathrm{H}_{2} \mathrm{O}+2 \mathrm{CO}_{2} \longrightarrow & \mathrm{Al}_{2} \mathrm{Si}_{2} \mathrm{O}_{5}(\mathrm{OH})_{4}+\mathrm{Ca}^{2+}+2 \mathrm{HCO}_{3} \\
\text { Anorthite } & \text { Kaolinite }
\end{array}
$$

L'abondance des bicarbonates dans les eaux de forage par rapport aux sources et puits est en accord avec le temps de séjour prolongé des eaux dans les aquifères de fissures et du caractère confiné de cet aquifère.
L'importance des ions $\mathrm{Cl}^{-}$et $\mathrm{NO}_{3}^{-}$dans les sources et puits est à relier à une origine anthropique et biogénique, et confirme que ces nappes sont plus superficielles. Ces ions proviendraient du pluviolessivage de la voûte forestière et des réactions d'oxydo-réductions de matières organiques liées aux activités humaines ou à la production animale ou végétale (Mathieu, 1976 ; Dakouré, 2003). La position superficielle des réservoirs captés par les sources et puits se traduit également par des valeurs élevées de turbidité qui est une mesure globale qui prend en compte toutes les matières, soit colloïdales, soit insolubles, d'origine minérale ou organique (Béchir et al., 2007)

En accord avec des indices de saturation négatifs des eaux vis-à-vis de la calcite et de la dolomie, et un équilibre avec la kaolinite, l'existence d'un bref temps de séjour et un taux de renouvellement important est confirmée pour les puits, mais encore plus pour les sources du fait de leur caractère plus superficiel. En revanche, les eaux de forages, qui appartiennent au domaine de la $\mathrm{Ca}$ montmorillonite à forte capacité de rétention, ont un temps de séjour plus long. Les travaux de Kamagaté (2006) sur les eaux d'altérites et de socle du bassin versant de la Donga ont abouti aux mêmes résultats. Par ailleurs, toutes les eaux se trouvent hors du domaine de l'albite. Ce qui indique soit un état de soussaturation ou de sur-saturation vis-à-vis de ce minéral. La similarité des caractéristiques hydrogéochimiques des eaux de puits et des eaux de sources suggère une origine superficielle des sources. Les sources seraient l'exutoire d'une nappe d'altérites plus superficielle que celle captée par les puits.

\section{Conclusion}

Cette étude, basée sur la composition géochimique des eaux souterraines et leur état d'équilibre dans les aquifères, montre que la signature chimique des eaux de sources naturelles du degré carré de Daloa est similaire à celle des eaux de puits captant les nappes d'altérites. Les eaux de sources et de puits sont faiblement minéralisées et plus récentes dans les aquifères que celles des forages. Ces caractéristiques montrent que les sources représentent des exutoires de nappes d'altérites. 
En plus des travaux de Maillary (1964), ces résultats constituent une étape préliminaire dans la connaissance du fonctionnement hydrogéologique des sources naturelles du degré carré de Daloa. La seconde étape de cette étude sera l'évaluation de la qualité microbiologique de ces eaux de sources consommées par les populations rurales.

\section{REMERCIEMENTS}

Nous voudrions remercier le Laboratoire Hydroscience de Montpellier (France) pour leur contribution aux analyses physico-chimiques. Nous remercions également la Société de Développement Minière de la Côte d'Ivoire (SODEMI) pour les données géochimiques mises à notre disposition.

\section{REFERENCES BIBLIOGRAPHIQUES}

Béchir BT, Khalifa R, Houda B. 2007. Elimination de la turbidité par oxygénation et filtration successives des eaux de la station de Sfax (Sud de la Tunisie). Rev. Sci. Eau, 20(4): 355-365.

Boukari M. 1982. Contribution à l'étude hydrogéologique des régions de socle de l'Afrique intertropicale : l'hydrogéologie de la région de Dassa-zoumé (Bénin). Thèse de doctorat, Université de Dakar, Dakar, p.147.

Bourlier JP, Lachassagne P, Desprats JF, Emmanuel G. 2005. Nouveaux éléments sur la structure et le fonctionnement hydrogéologique du plateau basaltique de l'aubrac (massif central, France). Première évaluation des potentialités en eau souterraine. C R. Géos., 337: 663673.

Canellas J. 1995. Au sujet de la définition et de la réglementation des eaux minérales naturelles. Houil. Bl., 2(3): 32-36.

Dakouré D. 2003. Etude hydrogéologique et géochimique de la bordure sud-est du bassin sédimentaire de Taoudéni (Burkina Faso - Mali) - essai de modélisation. Thèse de doctorat, Université Paris VI Pierre et Marie Curie, Paris, p.225.

Faillat JP, Grillot C. 1993. Différenciation hydrochimique de nappes superposées d'altérites et de fissure en socle granitique. J. Sci. Hydr., 38: 3-6.
Favara R, Grassa F, Inguaggiato S, D'Amore F. 1998. Geochemical and hydrogeological characterization of thermal springs in western Sicily, Italy. $J$. Volc. Geoth. Res., 84: 125-141.

Goné DL, Savané I, Yao NA, Biémi J. 2005. Mobilité relative des cations majeurs lors de l'altération des roches et acquisition de la minéralisation des eaux souterraines dans le degré carré de Man. Sci. Nat., 2(1): 85-94.

Goné DL, Savané I, Goblé MM. 2004. Caractéristiques physico-chimiques majeures des eaux des aquifères fissurées de la région d'Agboville (Sud-Ouest de la côte d'ivoire). Rev. ivoir. Sci. Technol., 5: 117-133.

Grillot JC, Ferry L. 1990. Approche des échanges surface-souterrain en milieu cristallin altéré aquifère (hautes terres de Madagascar). Cahier ORSTOM, Section Hydrol. Cont., 5(1): 3-12.

Kamagaté B. 2006. Fonctionnement hydrologique et origine des écoulements sur un bassin versant de milieu tropical de socle au bénin : bassin versant de la donga (haute vallée de l'ouème). Thèse de doctorat. Université de Montpellier 2, Montpellier, p.320.

Karakaya N, Karakaya MC, Nalbantçilar MT, Yavuz F. 2006. Relation between springwater chemistry and hydrothermal alteration in the Saplica volcanic rocks sebinkarahisar (Giresun, Turkey). J. Geoch. Expl., 93(1): 35-46.

Lasm T, Kouamé F, Oga MS, Jourda JRP, Soro N, Kouadio HB. 2004. Etude de la productivité des réservoirs fracturés des zones de socle. Cas du noyau archéen de Man-Danané (ouest de la côte d'ivoire). Rev. Ivoir. Sci. Technol., 5: 97-115.

Maillary JC. 1964. Notice explicative de la carte hydrogéologique à $1 / 200000$ de Daloa (Côte d'Ivoire). Contribution à la carte hydrogéologique de reconnaissance de la Côte d'Ivoire. BRGM-SODEMI, p.23.

Mathieu P. 1976. Influence des apports atmosphériques et du pluviolessivage forestier sur la qualité des eaux de deux bassins versants en Côte d'Ivoire. Cah. ORSTOM, Sér., Geol., 8(1): 11-32. 
Oi-wah L, Shiu-fai L. 2002. A survey on the composition of mineral water and identification of natural mineral water. Int. J. F. Sci. Technol., 37: 309-317.

Omar A A. 2008. Assessment of the spring water quality in the Shoubak area, Jourdan. Environ., 28: 203-215.

Papon A. 1973. Géologie et minéralisation du sud-ouest de la côte d'ivoire. Bulletin de la Direction des Mines et de la Géologie n ${ }^{\circ}$, Abidjan: 27.

Pouchan P. 1995. Emergence thermominérale. Houil. Bl., 2(3): 46-50.

Poulichet FE, Favreau G, Leduc C, Seidel JL. 2002. Major ion chemistry of groundwaters in the Continental Terminal
Water table of southwestern Niger (Africa). Appl. Geoch., 17: 1343-1349.

Schoeller H J. 1980. Influence du climat, de la température sur la teneur en $\mathrm{HCO}_{3}$ et $\mathrm{H}_{2} \mathrm{CO}_{3}$ des eaux souterraines. J. hydrol., 46: 365-376.

Soro N. 2002. Hydrochimie et géochimie et isotopique des eaux souterraines du degré carré de Grand-lahou et ses environs (sud-ouest de la côte d'ivoire). Implications hydrologiques et hydrogéologiques. Thèse, de doctorat, Université de Cocody, Abidjan, p 256.

Virgilio C, Zilda F. 2006. Hydrogeochemistry of thermal and mineral water springs of the Azores archipelago (Portugal). $J$. Volc. Geoth. Res., 151: 382-398. 